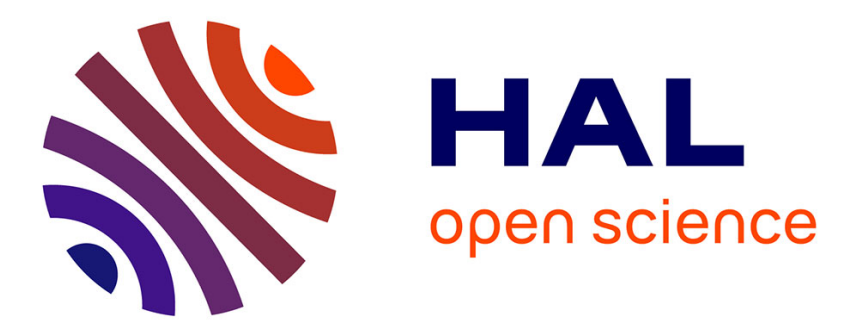

\title{
Numerically-aided 3D printed random isotropic porous materials approaching the Hashin-Shtrikman bounds
}

\author{
O. Zerhouni, M.G. G Tarantino, K. Danas
}

\section{To cite this version:}

O. Zerhouni, M.G. G Tarantino, K. Danas. Numerically-aided 3D printed random isotropic porous materials approaching the Hashin-Shtrikman bounds. Composites Part B: Engineering, 2019, 156, pp.344 - 354. 10.1016/j.compositesb.2018.08.032 . hal-01875669

HAL Id: hal-01875669

https://hal-polytechnique.archives-ouvertes.fr/hal-01875669

Submitted on 17 Sep 2018

HAL is a multi-disciplinary open access archive for the deposit and dissemination of scientific research documents, whether they are published or not. The documents may come from teaching and research institutions in France or abroad, or from public or private research centers.
L'archive ouverte pluridisciplinaire HAL, est destinée au dépôt et à la diffusion de documents scientifiques de niveau recherche, publiés ou non, émanant des établissements d'enseignement et de recherche français ou étrangers, des laboratoires publics ou privés. 


\title{
Numerically-aided 3D printed random isotropic porous materials approaching the Hashin-Shtrikman bounds
}

\author{
O. Zerhouni ${ }^{\mathrm{a}}$, M. G. Tarantino ${ }^{\mathrm{a}}$, K. Danas, ${ }^{\mathrm{a}, *}$ \\ ${ }^{a}$ LMS, C.N.R.S., École Polytechnique, 91128 Palaiseau, France
}

\begin{abstract}
The present study introduces a methodology that allows to combine 3D printing, experimental testing, numerical and analytical modeling to create random closed-cell porous materials with statistically controlled and isotropic overall elastic properties that are extremely close to the relevant Hashin-Shtrikman bounds. In this first study, we focus our experimental and 3D printing efforts to isotropic random microstructures consisting of single-sized (i.e. monodisperse) spherical voids embedded in a homogeneous solid matrix. The 3D printed specimens are realized by use of the random sequential adsorption method. A detailed FE numerical study allows to define a cubic representative volume element (RVE) by combined periodic and kinematically uniform (i.e. average strain or affine) boundary conditions. The resulting cubic RVE is subsequently assembled to form a standard dog-bone uniaxial tension specimen, which is 3D printed by use of a photopolymeric resin material. The specimens are then tested at relatively small strains by a proper multi-step relaxation procedure to obtain the effective elastic properties of the porous specimens.

Key words: Additive manufacturing, Hashin-Shtrikman bounds, Random Sequential Adsorption, Random composites, Homogenization, Porous Materials, Effective properties
\end{abstract}

\section{Introduction}

Porous materials are present in nature (e.g., rocks and geomaterials) but can also be designed to allow for controlled stiffness of lightweight structures. In the literature, one can recognize two main categories of porous materials; materials with closed-cell porosity, i.e. non-interconnecting voids, and open-cell porosity, which comprises most lattice and foam materials. In particular, this latter class of composites has been extensively studied in an effort to adapt the physical properties of their microstructure or micro-architecture (Fleck et al., 2010) by controlling morphological features of the internal geometry (Gibson and Ashby, 1997; Deshpande and Fleck, 2000). Such open-cell porous materials find applications in high-stiffness lightweight

\footnotetext{
* Corresponding author.

Email addresses: othmane.zerhouni@polytechnique.edu (O. Zerhouni), maria-gabriella.tarantino@polytechnique.edu (M. G. Tarantino), konstantinos.danas@polytechnique.edu (K. Danas,) 
structures (Zok et al., 2004; Berger et al., 2017), acoustic and vibration dampers (Göransson, 2006; Banhart et al., 1996; Ma et al., 2013), impact energy absorbers (Davies and Zhen, 1983), high electric capacitors (Wang et al., 2008) and filtration (Alderson et al., 2000) among others.

Specifically, in the context of open-cell porous materials, researchers have manufactured beam or metalsheet periodic cellular materials by electro-discharge machining Queheillalt et al. (2008) or clamping. Due to the strong manufacturing constraints, the design of controlled internal geometry was limited to simple geometries (Sypeck, 2005; Queheillalt et al., 2008). In contrast to conventional techniques, which are based on material removal, recent additive manufacturing technologies allow for the construction of microstructures layer-by-layer by use of various types of processes such as powder bed fusion (Heinl et al., 2007; Cansizoglu et al., 2008; Gorny et al., 2011), stereolithography (Hengsbach and Lantada, 2014) and photon lithography Meza et al. (2014) spanning scales from nanometer to meter.

The development of this new manufacturing process has led to the development of new methods for generating complex microstructures that can be 3D printed. One such popular approach is based on topology optimization methods among which is the ground approach (Bendsoe et al., 1994) that consists of finding the optimal structure using a discrete nodal description of the volume and minimizing a cost function of the boundary problem with a finite set of structural elements, usually taken as beams or voxels. Such materials are therefore optimal in a subset of microstructures resulting from that chosen element and the solution is dependent on the boundary conditions. Nevertheless, when using this methodology to create elastically-isotropic microstructures that can achieve extremal elastic properties and approach known bounds, for instance the Hashin-Shtrikman ones, such a task becomes less trivial. In particular, Sigmund (2000) has obtained such three-dimensional microstructures when assembling polyhedral regions connected by transversely isotropic three-rank laminates. The proposed microstructure, however, involves large scale variations and is therefore impossible to produce by current additive manufacturing techniques that only allow microstructures with similar length-scale orders.

Another approach, which is also popular in the literature, uses lattice network topologies obtained by connecting closest neighbors of crystallographic-like structures such as the Face-Centered-Cubic (FCC) Berger et al. (2017). These microstructures has been shown so far to deliver rather optimal mechanical properties in some specific directions due to the microstructural symmetries and under specific loads. In this context, Messner (2016), Meza et al. (2017) and Tancogne-Dejean and Mohr (2017) have recently designed almost-isotropic elastic microstructures by imposing a group of constraints on lattice truss networks. The resulting lattices were shown to lead to a fixed overall Poisson's ratio and a fixed relative effective Young's Modulus for a given porosity which is found in many cases to be relatively far from the upper HashinShtrikman bounds. Furthermore, the specific combination of the crystallographic-based microstructures proposed in order to fulfill the structural requirements have been numerically found by some of these authors to give an increasing deviation from the elastic-isotropy with increase of the relative density (i.e. decrease 
of porosity).

An alternative approach, that is at the heart of our present study, focuses on transferring theoretical and virtual microstructures that are very close to the Hashin-Shtrikman bounds numerically to fabrication and experimental testing. Contrary to the aforementioned lattice materials, most of the theoretical bounds are valid for closed-cell porous materials. Such works make use of minimization of energy principles which lead ultimately to rigorous theoretical bounds. Briefly, Hill (1952) derived the first bounds based on Voigt and Reuss estimates that respectively consider the strain and stress constant in the material. By introducing a reference linear elastic homogeneous material and a "constant" stress polarization in the microscopic equilibrium equations of linear elasticity, Hashin and Shtrikman (1963) and later Willis (1977) obtained rigorous bounds and estimates for the effective linear elastic behaviour for two- and N-phase isotropic and anisotropic materials, respectively. Such bounds are known to be optimal for isotropic two-phase microstructures as the effective bulk modulus is attained by the Hashin (1962) model of composite sphere assemblage (CSA) microstructure, whereby both effective shear and bulk moduli can be attained by the theoretical high-rank (6th rank) laminates (Francfort and Murat, 1986), as well as the dilated Poisson hyperplanes (Jeulin, 2001). Despite the great theoretical value of such microstructures, they inherently involve a large (or even infinite) range of length scales. This, in turn, does not allow as such their manufacturing with the current state-of-the-art 3D printing or other known manufacturing technology to-date.

This paper aims at introducing a methodology that allows to combine 3D printing, experimental testing, numerical and analytical modeling to create random closed-cell porous materials with statistically controlled and "isotropic" overall elastic properties that are extremely close to the Hashin-Shtrikman bounds. In this first study, we focus our experimental and 3D printing efforts to isotropic random microstructures consisting of single sized (i.e. monodisperse) spherical voids embedded in a homogeneous solid matrix. Those microstructures are shown to have almost identical effective elastic properties with multiple size (i.e. polydisperse) microstructures for volume fractions up to 30\% (see also Anoukou et al. (2018)).

In summary, Section 2 describes briefly the Random Sequential Adsorption (RSA) algorithm (Rintoul and Torquato, 1997; Segurado and Llorca, 2002; Böhm et al., 2002; Lopez-Pamies et al., 2013) used for generating virtual periodic microstructures and presents the numerical homogenization approach used to determine a representative volume element (RVE) allowing for rigorous comparison between analytical, numerical and corresponding experimental results independently of the applied boundary conditions (i.e. periodic or affine etc). Section 3 describes the process for assembling uniaxial tension specimens out of cubic porous RVEs and assesses the accuracy of printing with the aid of microscopy observations. Subsequently in the same section, an experimental setup is proposed to measure the linear elastic properties of the porous specimens by a multi-step relaxation procedure (Hossain et al., 2012). In Section 4, we examine the isotropy of the printing process for the pure matrix materials and discuss in detail the measurement sensitivity and effect of the support material used during the 3D printing to obtain the spherical voids. Subsequently, in 
Section 5, the effective experimental elastic properties of the porous materials are probed by finite element (FE) numerical estimates and the analytical Hashin-Shtrikman bounds. A contour analysis of the local stress and strain fields is used to interpret the influence of the microstructural features on the effective elastic properties. One of the important outcomes of this study is that the 3D printed porous material specimens are found to be very close to the theoretical Hashin-Shtrikmann upper bounds for porosities up to $30 \%$. Next, the numerical tools are used to study virtual microstructures with polydisperse (i.e. different size) voids for porosities up to $70 \%$ with very promising outcome. The study is concluded with Section 7 .

\section{Generation of random isotropic porous materials}

The particulate microstructures investigated in this study consist of a random distribution of nonoverlapping, identical spherical voids with volume fraction between $1 \%$ and $30 \%$. It is noted in passing that the methodology here presented is of general applicability and can be extended to deal with polydisperse spheres or ellipsoids (e.g. see works of Lopez-Pamies et al. (2013) and Anoukou et al. (2018)). In this section, we present briefly the approach used for the generation of the multi-inclusion systems with increasing porosity (the reader is referred to Segurado and Llorca (2002) and Lopez-Pamies et al. (2013) for a more detailed discussion)and then finite element (FE) simulations are carried out to define the size of the RVE necessary for the experimental analysis.

\subsection{RSA generation of isotropic random spherical inclusions}

In this section, we construct the virtual microstructures of the present study using the random sequential adsorption algorithm that was initially implemented for 3D microstructures by Rintoul and Torquato (1997). Following earlier work (see for instance Segurado and Llorca (2002) and Lopez-Pamies et al. (2013)), the centers of the spherical inclusions (here voids) are generated randomly in a cubic cell of volume $L^{3}$ and are rejected if the center-to-center distance to any of the already allocated voids is less than a given limit. To ensure an adequate finite element discretization, the minimum distance adopted is 1.02 times the average diameter of the two pores. Furthermore, since our microstructures are periodic, all voids that intersect the cubic cell faces have to be periodically reproduced to the opposite faces of the cube (see Segurado and Llorca (2002)). Finally, a pore that lies very close to the edge of the unit cell is also rejected if the distance between its center and one of the cell's faces is in the range of 0.95 and 1.05 times its diameter $D$. These two conditions are sufficient to obtain easy-to-mesh realizations. It is also worth noting that the parameters controlling the rejection or not of a newly placed void can be further reduced to allow the generation of larger volume fractions. However, the present study requires a minimum spacing between voids so that they can be realized properly by our 3D printer with a sufficient precision (see discussion in section 5 ). For illustration purposes, Fig. 1 shows three such RVEs comprising monodisperse spherical voids of increasing porosity $c=\{10,20,30\} \%$ with the number of pores $N$ used for their generation. 
a)

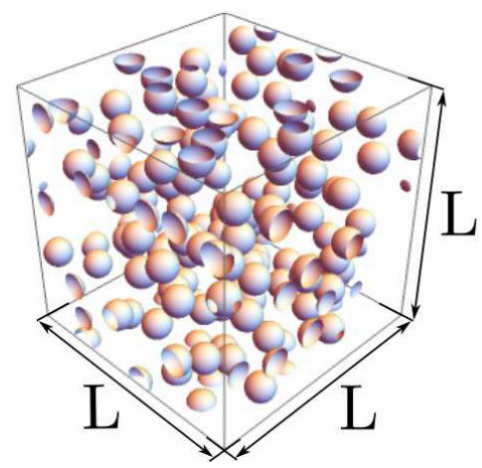

d)

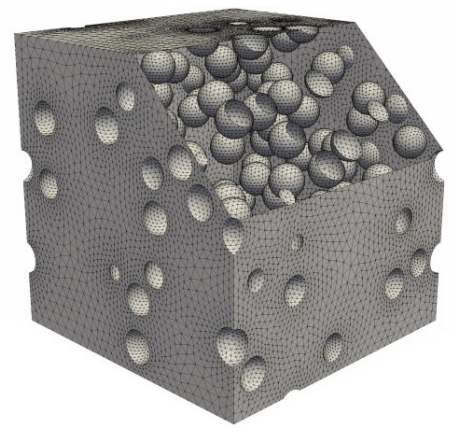

b)

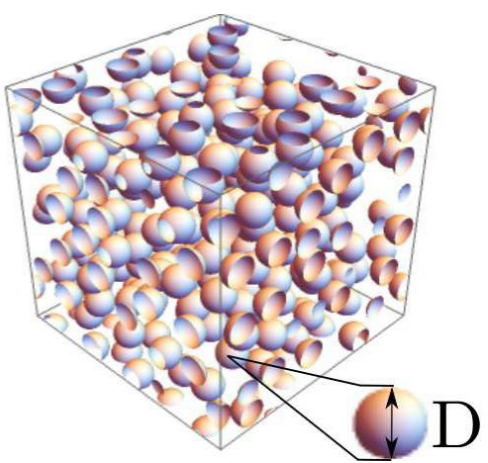

e)

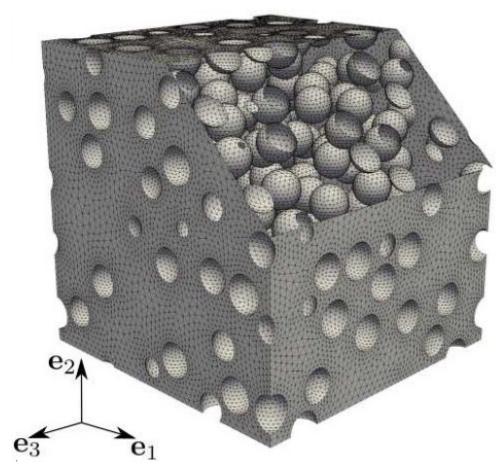

c)

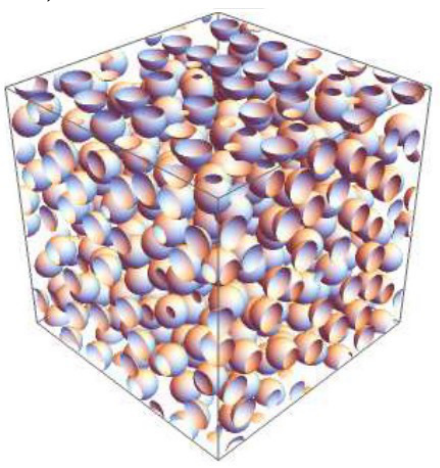

f)

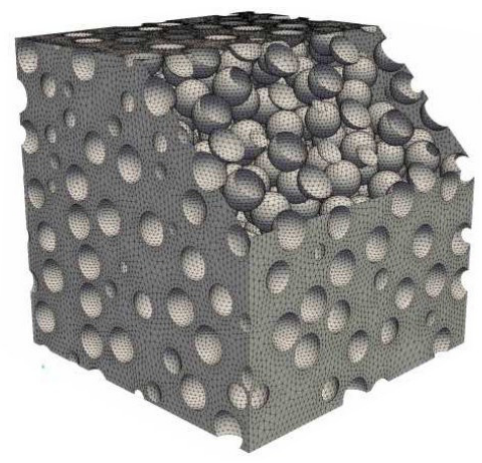

Figure 1: RVEs of unit volume $L^{3}$ with $N$ randomly distributed spherical particles of monodisperse sizes for a total porosity (a) $c=10 \%$ and $N=160$, (b) $c=20 \%$ and $N=275$, (c) $c=30 \%$ and $N=400$. (d-f) Representative meshes corresponding to the undeformed configuration of the representative cubic cells.

\subsection{RVE size and related boundary conditions}

The physical dimension of the cubic cell or equivalently the RVE size is directly related to the applied boundary conditions. Numerical studies on periodic unit-cells have shown (Suquet, 1987; Kanit et al., 2003) that the effective elastic properties of random particulate materials can be obtained with relatively small RVEs, i.e., for large values of the ratio $D / L$, with $D$ and $L$ denoting the diameter of the voids and the edge length of the cubic cell, respectively (see Fig. 1). For spherical voids and cubic RVEs, this ratio is given in terms of the number of voids $N$ and their volume fraction $c$ by

$$
c=\frac{N \pi}{6}\left(\frac{D}{L}\right)^{3}, \quad \text { or } \quad \frac{D}{L}=\sqrt[3]{\frac{6 c}{N \pi}} .
$$

Nevertheless, in uniaxial experiments as is the case in the following sections, the actual applied boundary conditions are not periodic (PBC) neither statically uniform (SUBC) nor kinematically uniform (i.e. average strain or affine) (KUBC). They are mixed SUBC-KUBC, i.e., part of the specimen is subjected to displacement control and another is stress-free. Therefore, we need to determine a cubic volume element 
that is representative for our subsequent uniaxial tension analysis. Following Suquet (1987), this RVE needs to have a sufficiently large number of voids, $N$, that behaves as if it were homogeneous in its bulk and exhibits only a small boundary layer that is affected by the non-periodic boundary conditions.

To address this non-trivial point, we define next our RVE by studying numerically two sets of boundary conditions, the periodic (PBC) and the uniform strain (KUBC) ones. The periodic boundary conditions are defined such that the displacement field $\mathbf{u}(\mathbf{x})$ is given by

$$
\mathbf{u}(\mathbf{x})=\bar{\varepsilon} \cdot \mathbf{x}+\mathbf{u}^{*}(\mathbf{x}), \quad(\mathrm{PBC})
$$

Here, $\bar{\varepsilon}$ denotes the average strain in the RVE, while $\mathbf{u}^{*}(\mathbf{x})$ is an $L$-periodic displacement field with zero volume average that accounts for the fluctuations of the field due to the inclusions/voids. For a technical discussion of how to apply such periodic boundary conditions, the reader is referred to Mbiakop et al. (2015) and Appendix B of Danas (2017).

Contrary to the $\mathrm{PBC}$, for which the homogenized elastic properties rapidly converge to the effective properties, the KUBC are known to be an upper bound for the apparent elastic properties of voided materials. Their deviation from the effective elastic properties is of the same order as the one obtained by using the SUBC (Huet, 1990). The KUBC consist in applying a uniform strain, such that

$$
\mathbf{u}(\mathbf{x})=\bar{\varepsilon} \cdot \mathbf{x}, \quad(\mathrm{KUBC})
$$

In the present study, convergence towards an experimental RVE is considered when (a) the effective properties obtained by the application of the KUBC reach an asymptotic value that is less than $2 \%$ from those corresponding to the PBC and (b) further increase of the number of pores does not affect the KUBC result by more than $0.1 \%$. For this purpose, a number of periodic cubic cells with gradually increasing number of voids are generated using a fixed porosity $c$. This is equivalent to decreasing the ratio between the characteristic size of the heterogeneities and the size of the cubic cell and $D / L$. For each pair $c$ and $N$, we create four different realizations to address the statistical deviation of the RSA process. Subsequently, the microstructures are discretized by use of ten-node tetrahedral quadratic elements (C3D10 in ABAQUS Version 6.11 (2011) software) using the automatic mesh generator NETGEN (Schöberl, 1997), which is also capable of creating identical surface meshes at opposite faces of the cell. This, in turn, allows for a direct implementation of the periodic boundary conditions by elimination techniques (e.g., use the *Equation command in ABAQUS).

\subsection{Definitions for isotropy}

The matrix phase is modeled by an isotropic linear elastic constitutive behavior with Young's Modulus $E=1.4 \mathrm{GPa}$ and Poisson's ratio $\nu=0.42$ corresponding to those of the virgin matrix phase used during the printing (a detailed discussion about the isotropy and properties of the matrix phase is done in Section 3.2). 
The homogenized stiffness tensor is computed from the average stress and strain fields using the overall constitutive equation (Hill, 1963)

$$
\langle\boldsymbol{\sigma}(\mathbf{x})\rangle=\widetilde{\mathbb{C}}:\langle\varepsilon(\mathbf{x})\rangle
$$

where $\langle\varepsilon(\mathbf{x})\rangle=\bar{\varepsilon}$ is the macroscopic imposed strain, $\widetilde{\mathbb{C}}$ denotes the overall stiffness tensor and the notation $\langle$.$\rangle is used to denotes volume averages. In practice, adopting Voigt notation, each column of the stiffness$ tensor $\widetilde{\mathbb{C}}$ is computed by imposing a strain field in a particular direction(Kanit et al., 2003). For example, imposing the overall strain field $\bar{\varepsilon} \equiv\left(\bar{\varepsilon}_{11}, 0,0,0,0,0\right)$, we obtain six linear relations: $\widetilde{C}_{k l 11}=\bar{\sigma}_{k l} / \bar{\varepsilon}_{11}$ with $k l=11,22,33,12,23,31$. The computation of all six columns of $\tilde{\mathbb{C}}$ is done by applying all six independent average strains, $\bar{\varepsilon}$, as described in Kanit et al. (2003).

Due to the finite number of inclusions, the tensor $\widetilde{\mathbb{C}}$ is not exactly isotropic. Therefore, one needs to estimate the resulting deviation from isotropy. To evaluate that deviation, an isotropic stiffness tensor, denoted $\tilde{\mathbb{C}}^{i s o}$, is introduced as a projection of $\tilde{\mathbb{C}}$ along the fourth-order deviatoric and hydrostatic tensors, i.e.,

$$
\widetilde{\mathbb{C}}^{i s o}=3 \tilde{\kappa} \mathbb{J}+2 \tilde{\mu} \mathbb{K} \quad \text { with } \quad \tilde{\kappa}=\frac{\widetilde{\mathbb{C}}: \mathbb{J}}{3}=\frac{\widetilde{C}_{i i j j}}{9}, \quad \tilde{\mu}=\frac{\widetilde{\mathbb{C}}: \mathbb{K}}{10}=\frac{3 \widetilde{C}_{i j i j}-9 \tilde{\kappa}}{30} .
$$

Here, $\tilde{\kappa}$ and $\tilde{\mu}$ denote the isotropized bulk and shear moduli, and $\mathbb{J}\left(J_{i j k l}=(1 / 3) \delta_{i j} \delta_{k l}\right)$ and $\mathbb{K}=\mathbb{I}-\mathbb{J}$ are the isotropic projection tensors, with $\mathbb{I}$ such that $I_{i j k l}=(1 / 2)\left(\delta_{i k} \delta_{j l}+\delta_{i l} \delta_{j k}\right)$, the identity tensor in the space of symmetric fourth-order tensors, and $\delta_{i j}$ (with $i, j=1,2,3$ ) the identity second-order rank tensor. Note that $\mathbb{J}$ and $\mathbb{K}$ satisfy the relations $\mathbb{J} \cdot \mathbb{J}=\mathbb{J}, \mathbb{K} \cdot \mathbb{K}=\mathbb{K}$, and $\mathbb{J} \cdot \mathbb{K}=\mathbb{K} \cdot \mathbb{J}=\mathbb{O}$.

There are two possible approaches for a quantitative measure of the deviation from isotropy; the geometrical approach which consists in evaluating the deviation from isotropy of the RVE in a geometrical sense (geometrical isotropy), and the mechanical approach, consisting in assessing the gap from isotropy of the mechanical response (mechanical isotropy), e.g. the effective stiffness tensor in the linear elastic context. In this study, we use the notion of mechanical isotropy since the goal is the evaluation of the effective elastic properties, and a deviation in geometric anisotropy (which is inherently present in our unit-cells) cannot be translated directly to deviation in mechanical isotropy.

Several authors (see for instance Zener and Siegel (1949), Spoor et al. (1995), Bucataru and Slawinski (2008), Moussaddy et al. (2013), Ghossein and Lévesque (2014)) have proposed methods which can be used to estimate the deviation from mechanical isotropy. They differ from each other by the measure of the amplitude of the stiffness tensor (represented in matrix form adopting Voigt notation), as well as the number of the coefficients used in that measure. The well-known measure of Zener's anisotropy ratio defined via $\delta_{z}=2 \widetilde{C}_{44} /\left(\widetilde{C}_{11}-\widetilde{C}_{12}\right)$ (using Voigt notation) takes only in consideration particular components of the stiffness tensor that correspond to cubic symmetry. Since our materials are random, we use instead the Frobenius norm as a measure of the amplitude of the stiffness matrix, which uses all components of $\widetilde{\mathbb{C}}$. The deviation from isotropy of $\widetilde{\mathbb{C}}$, denoted by $\delta_{\text {iso }}$, is then evaluated as a ratio of the Frobenius elastic distance 
function (Moakher and Norris, 2006)

$$
\delta_{i s o}=\frac{\left\|\widetilde{\mathbb{C}}-\widetilde{\mathbb{C}}^{i s o}\right\|_{F}}{\|\widetilde{\mathbb{C}}\|_{F}},
$$

where $\|\mathbb{A}\|_{F}=\sqrt{\operatorname{Tr}\left(\mathbb{A} \cdot \mathbb{A}^{T}\right)}$, is the Frobenius norm of the tensor $\mathbb{A}$. The value of $\delta_{i s o}=0$ corresponds to exact isotropy. The RSA algorithm is known to lead to very small deviation from isotropy when spherical inclusions are used (but see corresponding results for ellipsoidal inclusions in Anoukou et al. (2018)). In the present study, the maximum error is found to be $\delta_{i s o}<0.006$ and the deviation from $\delta_{z}=1$ which corresponds to the isotropic case is less than 0.01 . Therefore, the proposed RVEs can be considered isotropic.

\subsection{Converged RVEs}

Using the above definitions and boundary conditions, we carry out calculations to estimate the number of pores required for convergence in the effective elastic properties or equivalently the size of the RVE which is defined by the ratio $D / L$ in equation $(1)_{2}$. It is noted here that in addition to the converged RVE, a number of technical constraints related to the 3D printing accuracy and the experimental equipment have to be taken into account. Those constraints are discussed in detail in the following section. We recall that convergence towards an experimental RVE is considered when (a) the effective bulk and shear moduli obtained by the application of the KUBC reach an asymptotic value that is less than $2 \%$ from those corresponding to the $\mathrm{PBC}$ and (b) further increase of the number of pores does not affect the KUBC result by more than $0.1 \%$.

Specifically, Fig. 2 shows the evolution of the normalized effective moduli, defined as the ratio of the effective moduli of the RVE over the moduli of the matrix, for a porosity of $c=15 \%$ as a function of the number of pores $N$. The periodic boundary conditions (PBC) converge rapidly (i.e., for $N \sim 30$ ) to the effective elastic properties of the given microstructure and show no substantial dispersion among the various realizations. By contrast, the kinematically uniform boundary conditions (KUBC) require a much larger number of voids for convergence, in the order of $N=225$. This, in turn, leads to a size ratio $D / L \sim 0.103$, i.e. a side length of the cube that is approximately ten times the diameter of the void. Moreover, we observe that the KUBC loading leads to more dispersion among the different realizations.

Similar calculations, as those discussed in the context of Fig. 2 for porosity $c=15 \%$, are carried out for the entire range of porosities analyzed in this study allowing us to get a converged size ratio $D / L$ as a function of $c$, as shown in Fig. 3. We observe that $D / L$ increases with $c$ and is of the order $D / L \sim 0.1$. Overall a ratio $D / L \sim 9.85 \cdot 10^{-2}$ is sufficient for a converged RVE (green area in Fig. 3). This implies that the diameter of the voids should be less than $D \sim 1.18 \mathrm{~mm}$ for a cubic RVE of side length $L=12 \mathrm{~mm}$ in order to get converged effective elastic properties for any porosity $c$ (see right axis of Fig. 3). Instead, one can use larger void diameters at larger porosities $c$, as shown by the yellow area in Fig. 3 but it has to be taken case-by-case. The red regime, on the other hand, indicates non-converged $D / L$ ratios. Note at last that the converged ratios $D / L$ are also a function of the number of voids $N$ in the RVE, which are shown in the same figure as contours of equation $(1)_{2}$ (dotted lines). 

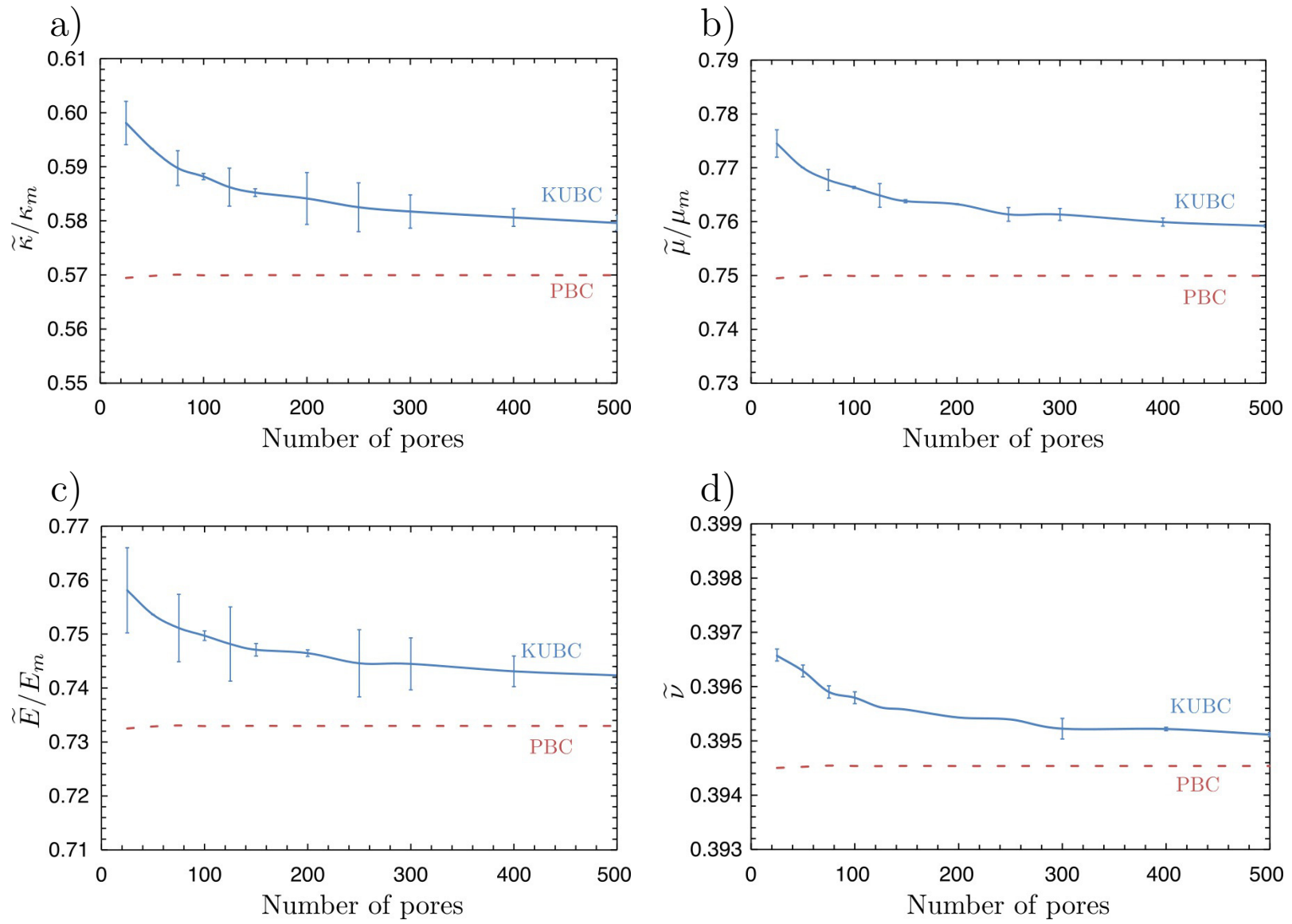

Figure 2: Numerical results to determine the number of monodisperse spherical pores that lead to convergence for the different boundary conditions PBC and KUBC. Normalized effective elastic moduli: (a) normalized bulk modulus $\widetilde{\kappa} / \kappa_{m}$, (b) normalized shear modulus $\widetilde{\mu} / \mu_{m}$, (c) normalized Young's modulus $\widetilde{E} / E_{m}$ and (d) Poisson's ratio $\widetilde{\nu}$ for $c=15 \%$. The matrix bulk, shear and Young's moduli are $\kappa_{m}, \mu_{m}$ and $E_{m}$, respectively.

\section{Additive manufacturing and experimental methods}

In this section, we describe the additive manufacturing procedure and experimental methods used to analyze the effective elastic properties of the pure matrix and porous materials. All test specimens are 3D printed using an acrylic photopolymer available in our EDEN 260VS 3D-printer purchased by Stratasys. The photopolymeric resin employed for 3D-printing has the commercial name VeroWhitePlus and is selected for its suitability to produce parts with very fine feature details, such as our spherical void microstructures. The main aim of the experiments is then the measurement of the effective Young's modulus and Poisson's ratio of the 3D-printed random porous microstructures. Due to the viscoelastic response of the VeroWhitePlus polymer, we measure the material parameters for the basic (ground state) elasticity by means of a tensile relaxation testing. Following the experimental methodology presented in Hossain et al. (2012), we carry 


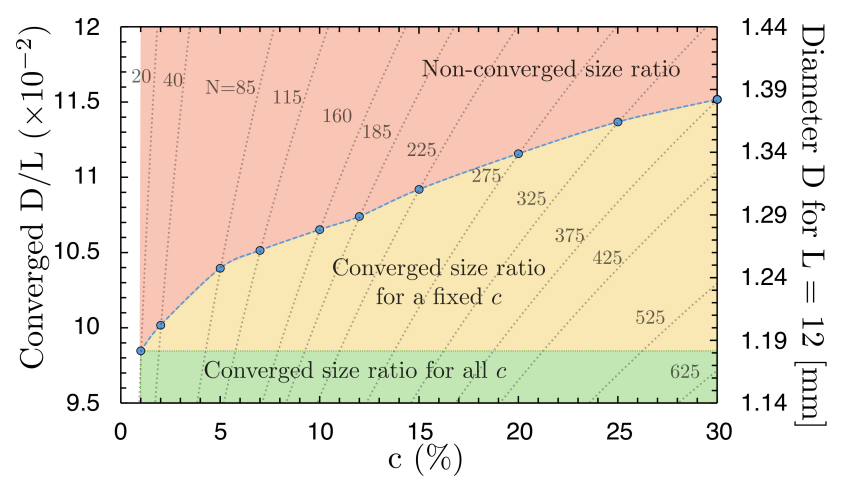

Figure 3: (Left axis) Converged in terms of elastic properties pore diameter-to-cube size ratio $D / L$ and (right axis) pore diameter (in $\mathrm{mm}$ ) for a cube side length $L=12 \mathrm{~mm}$ as a function of the porosity $c$. Colored regions depict in red the non-converged $D / L$ ratio, in yellow the converged $D / L$ ratio for given porosity and in green the maximum pore size, i.e., $D / L$ that can be used to obtain converged RVEs. Contours of equation $(1)_{2}$ for different number of pores $N$ are shown as dotted lines.

out two trains of experiments, namely (i) single- and (ii) multi-step relaxation tests, and we assess their suitability for obtaining the basic elasticity data from the time-dependent stress response.

\subsection{Additive manufacturing of porous specimens}

Specifically, our virtual test specimens have a dog-bone shape which is designed to ensure uniaxial stress conditions (on average since our porous specimens are heterogeneous) in the gage section. The ratio of the length to the width is for all samples higher than 10 (Wissler and Mazza, 2007). In order to construct the virtual geometry of the porous samples, we adopt the protocol shown in Fig. 4. We first assemble length-wise five representative cubic RVE cells of length $L=12 \mathrm{~mm}$ (see discussion in Section 3.2) in order to build the reduced uniform section of the test specimens. The latter is enclosed between the heads of the sample which, in turn, have a solid section thus allowing us to mount the specimen onto the uniaxial machine. The physical dimensions of the test specimens can be obtained from Fig. 4.

Next, we transform the 3D virtual model of our test specimens into a stereolitography format (i.e. STL) for 3D-printing. This is done by employing the commercial software NETGEN to mesh the 3D-models with four-node tetrahedral elements and subsequently export the latter to STL. We note in passing that more often than not the exported 3D STL models contain errors associated with the normal vectors defining the facets of the internal voids surfaces. In fact, these normal vectors define the direction towards which material should not be printed. Thus, one should correct the normal direction so that it points towards the center of the sphere. In the present study, those normal vector directions are corrected by use of the commercial software NETFABB (presently CadVision).

Once a correct STL model is produced, we then fabricate the specimens using the 3D-printer EDEN 


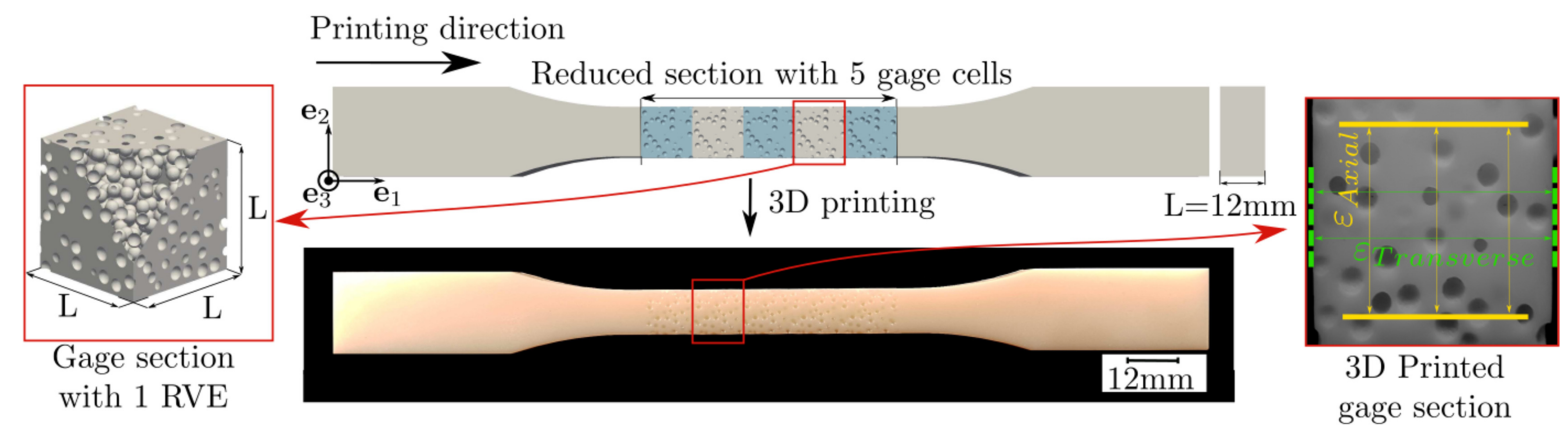

Figure 4: (Top) Virtual testing sample generated by assembling length-wise 5 cubic porous RVEs and by adding gripping heads of solid material. (Left) A zoom of the RVE which defines our gage section. (Bottom) 3D printed testing sample after cleaning support material from open pores and boundary surface. (Right) A zoom of the 3D printed RVE showing the size of the actual axial and transverse gage sections in the experiment.

260VS by Stratasys. Our printer employs a PolyJet technology that consists in building volume parts through layer deposition of liquid photopolymerizable droplets (of micrometric size) which are then cured with a UV light with no-additional post-curing. For the VeroWhitePlus resin used in this study, the layer resolution is $16 \mathrm{um}$ whereas that of the jetting precision is $100 \mu \mathrm{m}$, as specified by the manufacturer (for more information go to: http://www.stratasys.com). Furthermore, in order to overcome gravity constraints related to 3D objects such as that of a sphere, our 3D-printer uses a support gel-like material to print the spherical void volumes during layer deposition. As we will show in Section 4.3, this support material has no influence on the measured effective response of the porous composites. Moreover, it is non-toxic and is easily removed from the specimens outer surface by water-jet cleaning after manufacturing.

a)

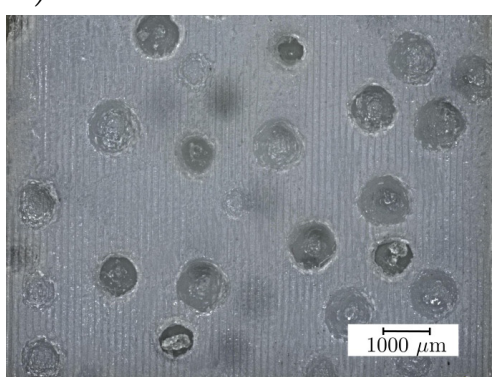

b)

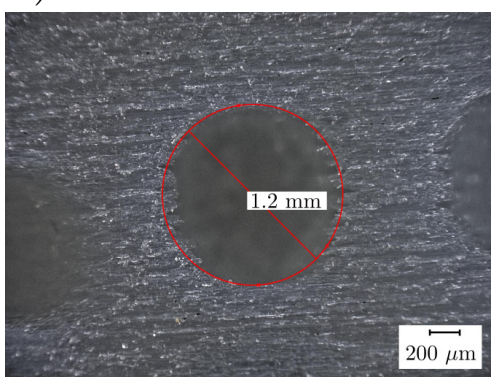

c)

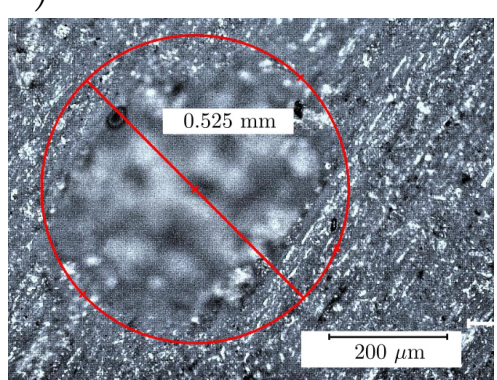

Figure 5: Optical image showing a cross-section of the macroscopic 3D printed specimen with the spherical pores for the assessment the printing accuracy. Observations of representative 3D printed spherical voids of diameter (b) $D \sim 1.2 \mathrm{~mm}$ and (c) $D \sim 0.500 \mathrm{~mm}$. A fairly good printing accuracy is observed in both cases.

We assess the dimensional accuracy of the 3D-printed porous microstructures by use of an optical microscope. Specifically, guided by the results of the numerical RVE calculations in Fig. 3, we have investigated 
several void diameters spanning from $D=400 \mu \mathrm{m}$ to $D=1200 \mu \mathrm{m}$. In order to observe the microstructures under the microscope, we deliberately interrupted the 3D-printing process at arbitrary time steps and then resumed it after optical analysis. A set of representative optical micrographs are shown in Fig. 5, with parts (b) and (c) corresponding to pore diameters of $\sim 1.2 \mathrm{~mm}$ and $\sim 0.5 \mathrm{~mm}$, respectively. Overall, Fig. 5 reveals that pores with circular section are finely fabricated in both cases and can be manufactured with very good dimensional accuracy within the range of size explored. In connection with Fig. $3, D \sim 1200 \mu \mathrm{m}$ corresponding to Fig. 5a,b is the minimum pore size required by our numerical RVE analysis for $L=12 \mathrm{~mm}$, as discussed in Section 2.4. Finally, it is worth mentioning that no additional micron-size porosity has been observed in the matrix phase as a result of the printing process.

\subsection{Experimental protocol}

The tensile relaxation experiments are carried out at room-temperature using a servo-hydraulic uniaxial apparatus. We measure the load history with a $10 \mathrm{kN}$ force transducer (accuracy $\pm 10 \mathrm{~N}$ ). In turn, the axial and transverse strains are obtained simultaneously and independently by means of two clip-on gage extensometers. Specifically, the former is measured with an MTS $632.13 \mathrm{~F}-20$ sensor (accuracy $\pm 0.0075 \mathrm{~mm}$ ) and the latter with an Epsilon 3475-025M-ST transducer(accuracy $\pm 0.1 \mathrm{~mm}$ ). It is important to note here that since we are interested in measuring the effective properties of the porous materials, the gage length for each extensometer was set equal to the characteristic cubic RVE cell length, i.e. $L=12 \mathrm{~mm}$ (see discussion in Section 2). It is not the goal of the present study to measure the local strains in-between the voids or any other local information.

For consistency with the numerical analysis, for each test, we use four specimens to assess the degree of reproducibility of the experimental results. We also note that in order to minimize the discrepancy of the measurements, every test sample is printed individually and experiments are conducted three days after the manufacturing process (to prevent aging of the polymer matrix). As reported by Barclift and Williams (2012), the relative distance between the samples as those are set upon the building tray of the 3D-printer could be an additional source of scattering in their elastic properties.

\subsubsection{Single relaxation testing}

A common approach in determining the time-independent equilibrium response, i.e., the equilibrium stress state, in viscoelastic polymers is to perform single-step relaxation tests under a constant applied displacement. Such tests typically involve the application of a constant displacement (leading to a constant overall strain) and then monitoring the force decay over time.

The value that the stress reaches asymptotically at the end of the holding time in single-step relaxation experiments corresponds to the equilibrium stress. Therefore, it is the value of interest for the estimation of the Young's modulus. We assess the suitability of this testing method by carrying out three relaxation tests 
at different strain rates $^{1} \dot{\varepsilon} \equiv \dot{\bar{\varepsilon}}=\left\{10^{-4}, 10^{-5}, 10^{-6}\right\} \mathrm{s}^{-1}$ for the pure VeroWhitePlus material (i.e. without voids) and by measuring the load history under an applied constant nominal strain of $\sim 0.5 \%$. Those singlestep relaxation tests, albeit useful to obtain the relaxation decay of the material and an approximate range of the linear response regime, are inappropriate to provide the pure (i.e. without viscoelastic contributions) elastic modulus of the material.

\subsubsection{Multi-step relaxation testing}

In view of this, multi-step relaxation tests at various levels of deformation is an alternative to singlerelaxation and can be employed to determine the basic elasticity (slopes of the stress-strain response) of the materials under study. In many practical cases, and particularly when the amount of the viscous effects in the material are unknown, the method proves more suitable and time-effective than the single-step relaxation as discussed by Hossain et al. (2012). In the present study, multi-step relaxation tests at $\dot{\varepsilon}=10^{-5}$ are conducted. At each step a displacement corresponding to an average axial strain increment of $0.1 \%$ is applied to the test specimen and the step-wise load history is recorded. At each step, the holding time for relaxation is variable. Specifically, we consider the material to be at its equilibrium state, and therefore attaining its purely elastic response, when the difference between two consecutive force measurements, taken at 20 min-time intervals, is smaller than $10 \mathrm{~N}$ (which corresponds to the accuracy of our load cell). Moreover, the tests are interrupted at a nominal strain of $\bar{\varepsilon}=0.7 \%$ and hence each test consists of seven relaxation steps. As we will show in the next section, beyond this value of the nominal strain, the stress-strain response of the polymer VerowhitePlus matrix starts departing from linearity.

\section{Experimental results for the matrix and support materials}

In this section, we show detailed experimental results for the matrix and support material following the procedure discussed in the previous section. The experimental protocol is exactly the same for the porous specimens and is not discussed independently.

\subsection{Elastic moduli of the monolithic VeroWhitePlus matrix}

Experimental results of the single-step relaxation tests for the monolithic VeroWhitePlus matrix at different strain rates $\dot{\bar{\varepsilon}}=\left\{10^{-4}, 10^{-5}, 10^{-6}\right\} \mathrm{s}^{-1}$ are reported in Figs 6a,b. Data in Fig 6a provide quantitative measurements of the matrix strain rate sensitivity as well as of its time-dependent response. The comparison of the curves in Fig 6a shows that the initial slope of the axial force-time response decreases with decreasing strain rate, whereas not all three curves reach to the equilibrium state within the holding time window,

\footnotetext{
${ }^{1}$ Henceforth the () notation used to denote average measures is dropped for simplicity and illustration purposes unless otherwise stated.
} 
although they all exhibit the tendency to reach the same asymptotic force value at large times. For completeness, we report in the inset of Fig 6a the measured stress-strain response of the matrix at two different strain rates $\dot{\bar{\varepsilon}}=\left\{10^{-5}, 10^{-6}\right\} \mathrm{s}^{-1}$ and observe that they depart from linearity after $\sim 0.7 \%$ strain. Therefore, in order to obtain the initial Young's modulus one has to make sure that the overall applied strains remain small, which makes experimental measurements extremely sensitive. On the other hand, Fig $6 \mathrm{~b}$ reveals that the Poisson's ratio, obtained as the absolute value of the ratio between the transverse strain over the axial strain, is strain-rate insensitive.

a)

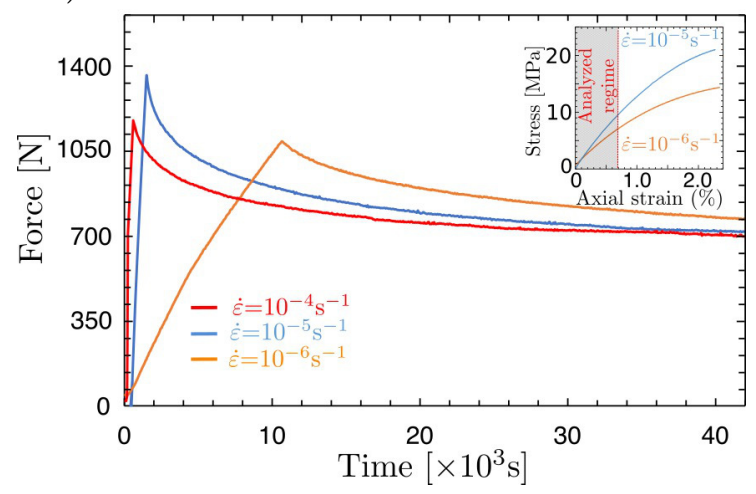

b)

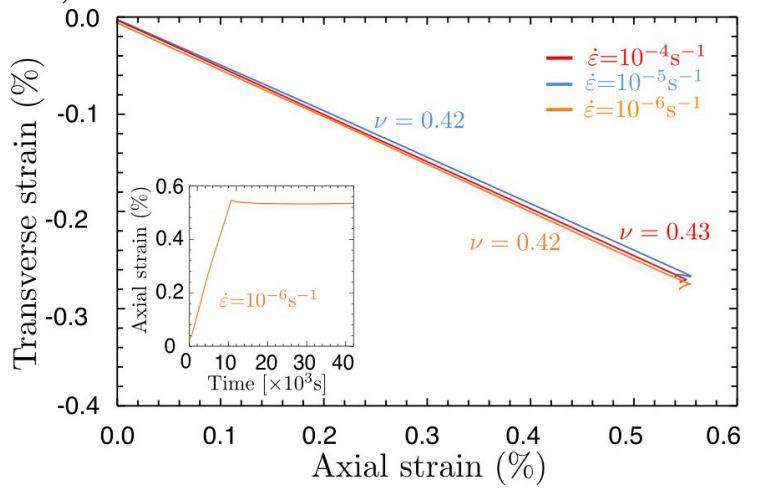

Figure 6: (a) Force measurements for single-step relaxation tests at different strain rates showing strong ratedependence. Inset shows the corresponding axial stress-strain response for strain rates $\dot{\bar{\varepsilon}}=\left\{10^{-5}, 10^{-6}\right\} \mathrm{s}^{-1}$. Axial versus transverse strain curve obtained for different strain rates showing that the Poisson's ratio is fairly rate-independent. Inset shows the evolution of the applied strain as a function of time.

a)

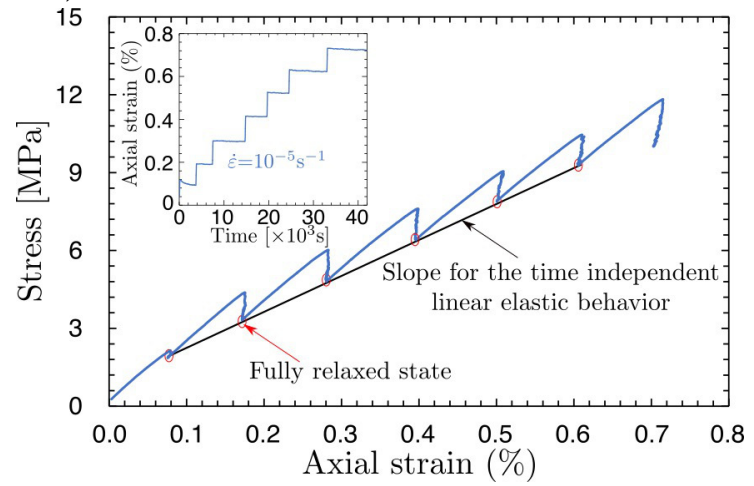

b)

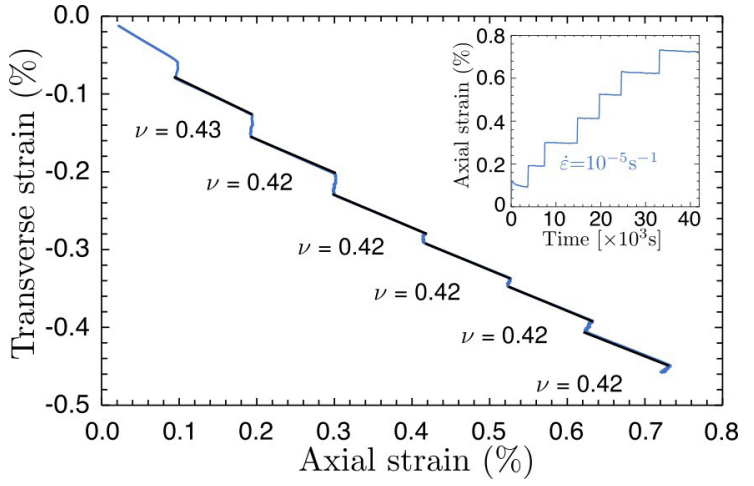

Figure 7: Overall applied strain rate $\dot{\bar{\varepsilon}}=10^{-5} \mathrm{~s}^{-1}$ : (a) Stress-Strain curves obtained during multi-step relaxation steps. The Young's modulus is obtained by the slope of the curve that connects the fully relaxed stress states excluding the first point. (b) Axial versus transverse strain curve during the multi-step relaxation test. Slopes during loading are found to be fairly independent of the level of the axial strain. 
The results of the multi-step relaxation experiments at $\dot{\bar{\varepsilon}}=10^{-5} \mathrm{~s}^{-1}$ for the VeroWhitePlus matrix are reported in Fig 7a. The effective Young's modulus, $\widetilde{E}$ (or $E_{m}$ for the pure polymer), is then evaluated as the slope of the line connecting the equilibrium (fully relaxed) stress points in the nominal stress-strain curve as shown in Fig. 6a. It is worth mentioning that in the evaluation of the slope, we use six equilibrium stress points, neglecting the one at zero strain, which can be very sensitive to initial settings of the experimental setup (e.g., gripping of the specimen, minor sliding between the heads and the machine and sensitivity of the extensometers). Moreover, in agreement with previous observations in Fig 6b, Fig. 7 shows that at each relaxation step the slope of transverse strain-axial strain curve is time-independent thereby making the calculation of the Poisson's ratio straightforward. Finally, a very small drop is observed for the transverse strain at each relaxation step.

\subsection{Isotropy of the $3 D$ printed matrix material}

Prior to testing the porous materials, we first investigate experimentally the isotropy of the mechanical response of the 3D-printed pure VeroWhitePlus matrix. This analysis is extremely important for our study since the microstructural anisotropy (if any) could be easily attributed to the intrinsic anisotropy of the polymer matrix. In order to address this issue, we print the dog-bone specimens in all three different directions with respect to the printer heads. For clarity, hereinafter $\mathbf{e}_{1}$ corresponds to the printing direction, i.e. the displacement direction of the printer's heads, $\mathbf{e}_{2}$ and $\mathbf{e}_{3}$ are respectively the in-plane and out-ofplane directions perpendicular to $\mathbf{e}_{1}$. We also note that $\mathbf{e}_{3}$ corresponds to the direction of deposition of the sequential layers. In Fig. 8, we report computed values of the Young's modulus and Poisson's ratio

a)

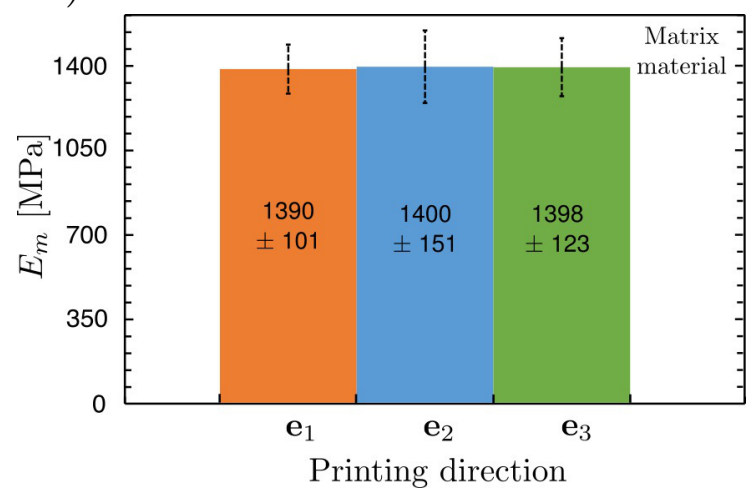

b)

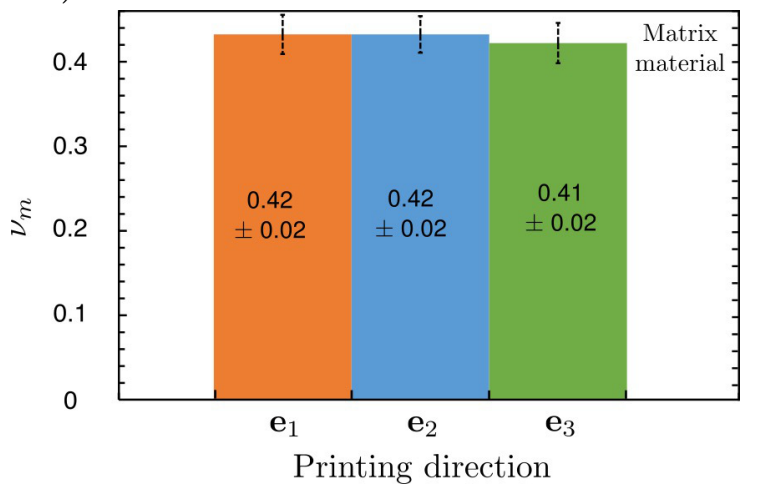

Figure 8: Experimental results of (a) Young's modulus $E_{m}$ and (b) Poisson's ratio $\nu_{m}$ for the matrix phase with respect to the printing direction. The matrix is found to be isotropic.

for all three different printing directions. The elastic parameters are obtained from multi-step relaxation experiments as described in the previous section. As seen, the measured values of these moduli are within the scatter of data and are independent of the printing direction. The measured mechanical isotropy can 
partially be explained by observation of Fig 5a. In our 3D printer, the liquid polymer droplets create a homogeneous solid material when coalescing and overcome any apparent laminated microstructure that would result from the $16 \mu \mathrm{m}$ layering process. Finally, our data provided experimental evidence that the matrix material (VeroWhite) is statistically homogeneous and exhibits an elastically isotropic behavior with a Young's modulus $E_{m}=1400 \pm 120 \mathrm{MPa}$ and a Poisson's ratio of $\nu_{m}=0.42 \pm 0.02$. These moduli correspond to a bulk modulus of $\kappa_{m}=2920 \mathrm{MPa}$ and a shear modulus of $\mu_{m}=493 \mathrm{MPa}$.

\subsection{Effect of support material}

This section deals with the influence of the gel-like support material used to overcome gravity issues while printing the internal spherical void geometry. As anticipated, the support material can be easily removed from the external surfaces of the 3D-printed parts by waterjet or chemical NaOh solution if it is inside an open (or connected with the surface) porosity. For closed-cell porosity, as is the case in the present study, this material is trapped inside the structure. Thus, the mechanical characterization of the proposed porous

a)

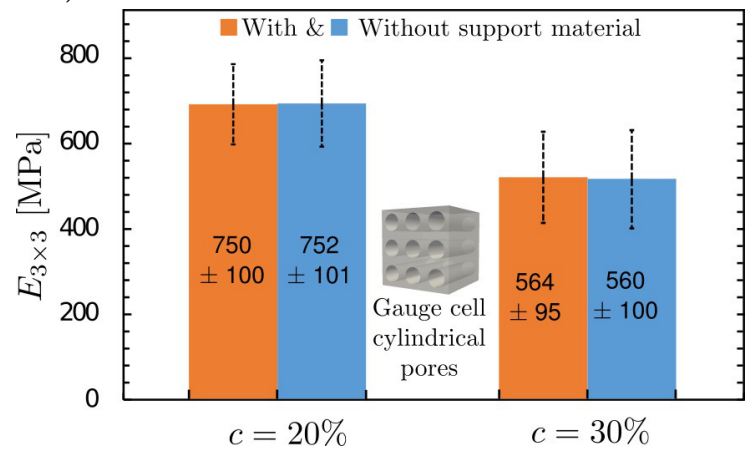

b)

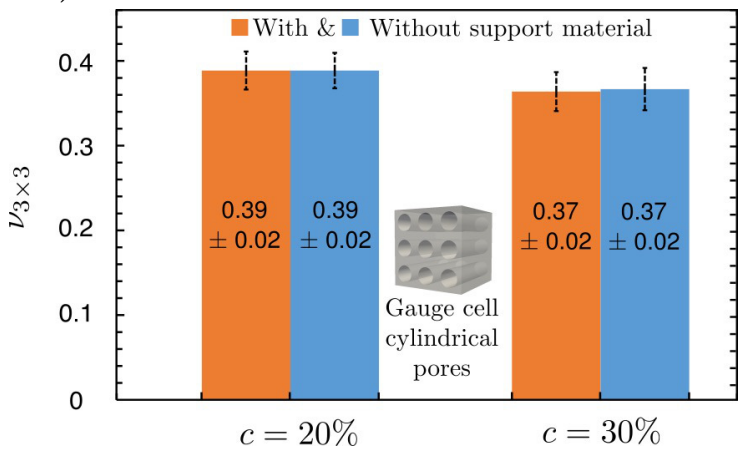

Figure 9: Experimental results of the apparent (a) Young's modulus and (b) Poisson's ratio for the investigation of the effect of the support material inside the voided phase. A cylindrical-void specimen is specifically fabricated in order to control the presence or not of the support material. The support material has negligible effect upon the elastic properties.

materials needs to account for the effect of this support material and examine if it behaves closely to a voided material. To that aim, specimens with gage zone consisting of aligned cylinders in the direction of the specimen thickness (see insets in 9) are printed and characterized in two configurations; one where the support material has been removed from the cylinders and the other one where the support material has been retained. Results of the apparent overall Young's modulus ${ }^{2}$ for these two structures are shown in Fig. 9. No significant difference is found between the two configurations, indicating that the support material

\footnotetext{
${ }^{2}$ Note that these structures are not representative and hence the measured elastic properties are those corresponding to a structure and not a material.
} 
has negligible elastic properties when compared with those of the virgin matrix material (VerowhitePlus). This implies that the inclusions can be safely considered as voids in the remaining of the study.

\section{Results for porous materials}

In this section, we compare the theoretical Hashin-Shtrikman (HS) bounds for isotropic porous materials, with numerical (FEM) calculations and the present experimental measurements. Since, the proposed microstructures exhibit almost perfect isotropy by construction, direct comparison of their elastic effective properties with the HS isotropic bounds is meaningful in terms of moduli, such as the Young's modulus and Poisson's ratio or equivalently the bulk and shear moduli. For isotropic porous materials, the HashinShtrikman bounds (Hashin and Shtrikman, 1963; Willis, 1977) are given by

$$
\widetilde{\kappa}_{H S}=\frac{4(1-c) \mu_{m} \kappa_{m}}{4 \mu_{m}+3 c \kappa_{m}} \quad, \quad \widetilde{\mu}_{H S}=\frac{(1-c)\left(8 \mu_{m}+9 \kappa_{m}\right) \mu_{m}}{4 \mu_{m}(2+3 c)+3 \kappa_{m}(3+2 c)}
$$

where $\kappa_{m}$ and $\mu_{m}$ denote the bulk and shear moduli of the matrix phase, respectively and $c$ the volume fraction of the inclusion phase or porosity in the present context. The corresponding effective Young's modulus, $\widetilde{E}$ and Poisson's ratio, $\widetilde{\nu}$, are readily obtained by $\widetilde{E}=9 \widetilde{\kappa} \widetilde{\mu} /(3 \widetilde{\kappa}+\widetilde{\mu})$ and $\widetilde{\nu}=(3 \widetilde{\kappa}-2 \widetilde{\mu}) /(6 \widetilde{\kappa}+2 \widetilde{\mu})$, respectively. It is recalled at this point that the HS homogenization bounds refer to infinitely polydisperse (i.e. infinite sizes of) voids. Nonetheless, as we will see later in Fig. 6, for porosities up to $c=30 \%$ (see also Anoukou et al. (2018)), the monodisperse and polydisperse results are almost identical. Moreover, we recall here that both the FEM and experimental results correspond to four different realizations and samples, respectively, for each porosity.

Figure 10 shows the effective (a) Young's modulus $\widetilde{E}$, (b) Poisson's ratio $\widetilde{\nu}$, (c) bulk modulus $\widetilde{\kappa}$ and (d) shear modulus $\widetilde{\mu}$ as a function of the porosity $c$. We observe that the experimental results for the directly measured $\widetilde{E}$ are in very good agreement with the numerical FE results, which almost overlap with the HS bounds even for porosities as large as $c=30 \%$. The maximum deviation of the experimental $\widetilde{E}$ from the HS bound is $8 \%$ when $c=30 \%$. Note, however, that the experimental scatter has a similar range (i.e. in the order of $8 \%$ ) for the various generated porosities and statistical samples suggesting that a main factor for this dispersion is the conditioning of the polymeric material during 3D printing as well as measurement sensitivity resulting from the extensometers. It is noted that the FE results exhibit very small deviation (less than $0.1 \%$ ), and thus the corresponding scatter is not shown explicitly in the plots. In turn, the scatter in the experimental results of the Poisson's ratio is larger and can be attributed principally to the sensitivity of the extensometer. The effective bulk $\widetilde{\kappa}$ (Fig. 10c), and shear, $\widetilde{\mu}$ (Fig 10d), moduli are computed from the mean value of the Poisson's ratio and Young's modulus whereas their scatter is evaluated using the scatter

of the Young's modulus measures only. Similar to $\widetilde{E}$, both the $\widetilde{\kappa}$ and $\widetilde{\mu}$ obtained by the experiments are very close to the theoretical HS bounds. 

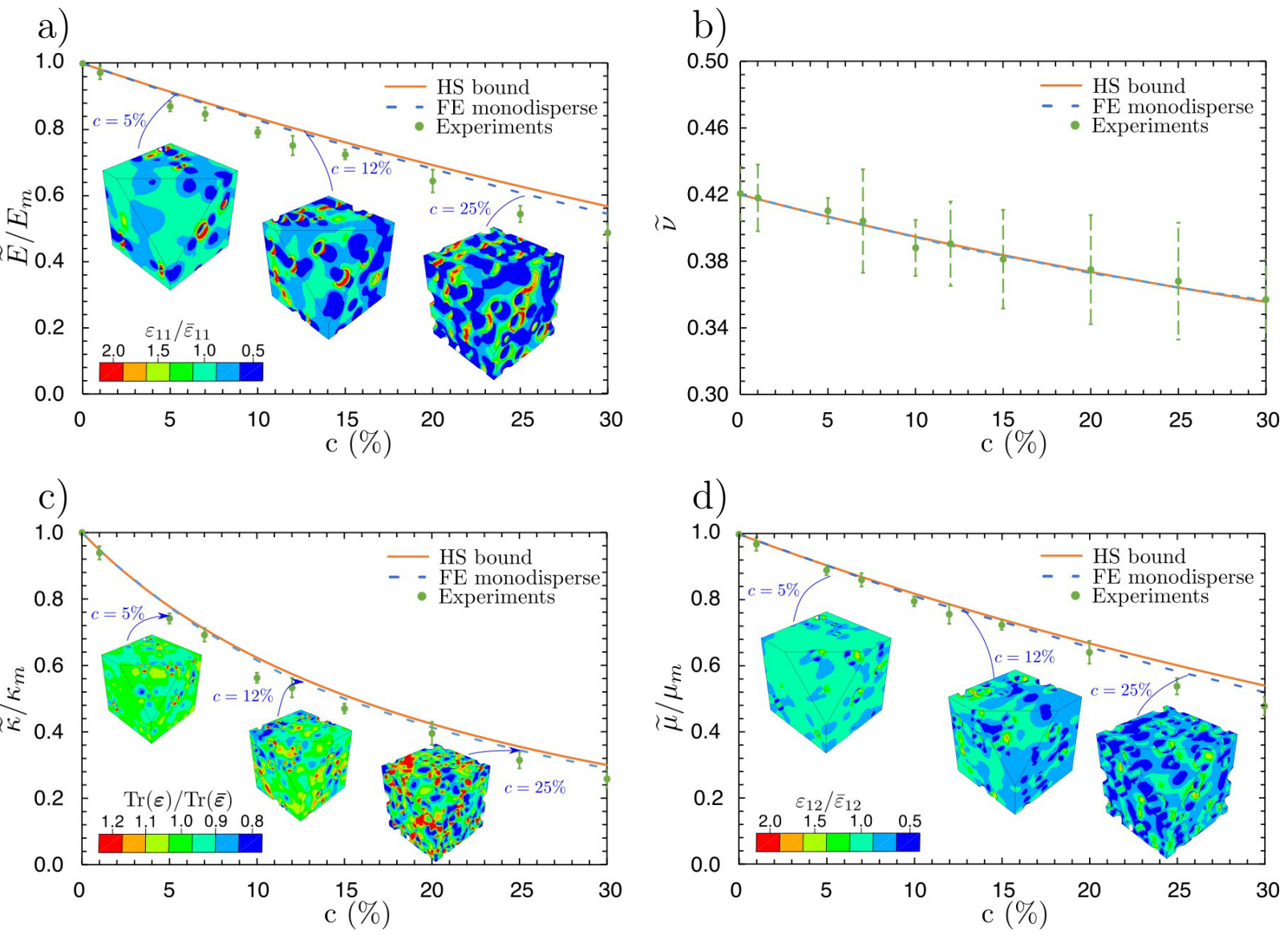

Figure 10: Normalized with the matrix properties effective (a) Young's modulus $\widetilde{E} / E_{m}$, (b) Poisson's ratio $\widetilde{\nu}$, (c) bulk modulus $\widetilde{\kappa} / \kappa_{m}$ and (d) shear modulus $\widetilde{\mu} / \mu_{m}$ as a function of the porosity $c$. Comparison between the analytical HS bounds, FE monodisperse numerical estimates and experimental results.

It is relevant at this point to make a few important comments. In particular, we recall that the HashinShtrikman bounds are obtained by setting a constant stress polarization in the inclusion phase (Hashin and Shtrikman, 1963; Willis, 1977), which implies uniform stress and strain fields therein. That allows to obtain the Eshelby (1957) exact solution for a dilute volume fraction. Nevertheless, this choice of uniform stress polarization is only approximate for spherical or ellipsoidal inclusions and moderate to high volume fractions (Willis, 1977). This has the following two implications. First, spherical voids cannot reach, even numerically, the HS bounds with increasing porosity, since the fields cannot remain uniform inside the inclusions due to strong interactions between them. Nevertheless, it is more than interesting to observe in Fig. 10 that both the experimental and numerical effective elastic moduli for spherical voids remain very close to the theoretical HS bounds. Second, we recall that the 6-rank laminate microstructures (Francfort and Murat, 1986) and the infinite rank-laminates (Idiart, 2008) do attain the HS bounds primarly because the fields inside each phase of the microstructure are uniform and thus are in line with the constant sress 
polarization assumption used in the HS bounds. It is however difficult to-date to reproduce realistically such microstructures due to the many length scales involved in their construction (but see Sigmund (2000) towards this direction).

We close this section by a brief discussion of the local fields obtained by our FE calculations. Specifically, we show in the insets of Fig. 10 strain contours for selected porosities $c=\{5 \%, 12 \%, 25 \%\}$. The uniform distribution of voids avoids clustering and leads to rather minimal stress and strain concentration in regions between the voids. For porosities up to $10 \%$, the local strain fluctuations induced by the microstructural heterogeneities remain relatively small by comparison to the overall applied strain $\bar{\varepsilon}$ (see colorbars). When increasing the porosity, the denser packing of the voids creates more localized strain zones with more pronounced strain fluctuations with respect to the average strain. This implies significant interactions between voids, which, in turn, lead to the deviation observed between the numerical results and the theoretical HS bounds. Nevertheless, this deviation remains very small $(<4 \%)$ (see also Böhm and Han (2001)).

Finally, in connection with those last observations, it is important to mention that in the FE calculations the local strains can reach values that are almost twice that of the average strain applied, see for instance $\varepsilon_{11} / \bar{\varepsilon}_{11} \sim 2$ in the inset of Fig. 10a. This, in turn, implies that in the experimental results and usually for porosities higher than $20 \%$, the amplitude of the local strain fields could exceed the range of validity of linear elasticity in several regions of the unit-cell. This leads to a local nonlinear response of the matrix phase, especially in our experiments (see inset of Fig. 6a), which constitutes an additional reason for the differences observed between the HS linear bounds or the FE and the experimental measurements, especially for large porosities.

\section{Estimates for higher volume fraction with polydisperse spheres}

In this section, we study numerically and analytically the possibility to extend the present study to larger porosities, at least up to $c=70 \%$ (or relative density $\rho=1-c=0.3$ ). That is impossible to achieve with monodisperse distributions of voids, since they exhibit naturally a interpenetration limit near $c \sim 64 \%$ (Torquato, 2002). Nevertheless, using the present RSA algorithm for polydisperse (i.e. various sizes of) voids (see works of Lopez-Pamies et al. (2013) and Anoukou et al. (2018)), one can reach higher volume fraction rather effortless.

Figure 11 shows such three representative periodic RVEs comprising random polydispesrse voids in volume fractions (a) 35\%, (b) $50 \%$ and (c) $70 \%$. For porosities up to $c=60 \%$, we have used seven different families (or sizes) of voids with a relative size ratio between the smallest and the largest one set at a value of 2.5. Beyond that point, we use ten different families of void sizes with maximum relative size ratio of 10. Currently, the higher porosity RVEs lead to minimum void sizes that reach the limits of our additive manufacturing capabilities and hence are not presented here but are left for a future study. 
a)

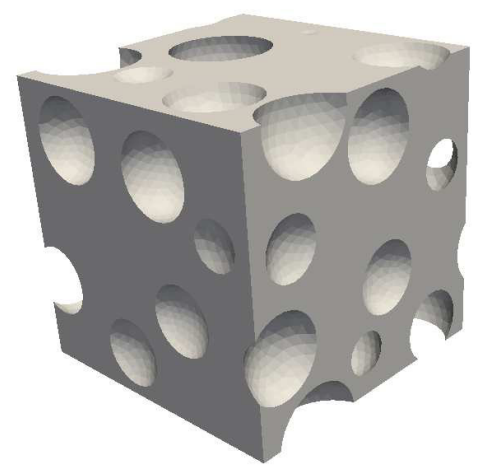

b)

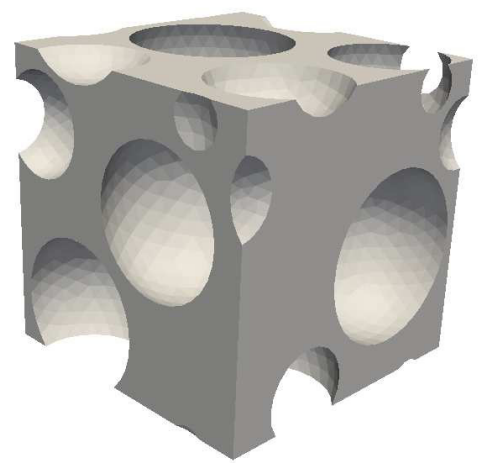

c)

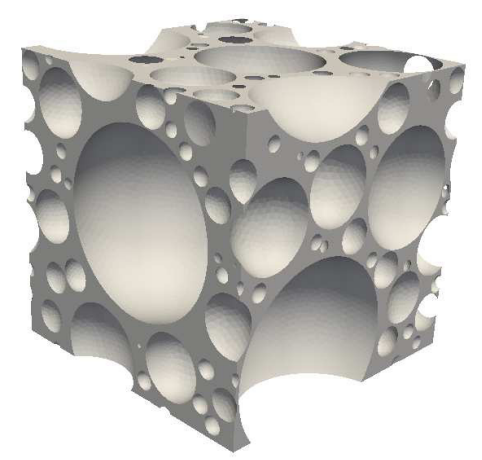

Figure 11: Periodic cubic cells of volume $L^{3}$ with $N$ random polydisperse spherical voids and porosity (a) $c=35 \%$ with 7 families and maximum size ratio $\left(S R_{\max }\right)$ of $S R_{\max }=1.50$, (b) $c=50 \%$ with 7 families and $S R_{\max }=2.50$, (c) $c=70 \%$ containing 10 families of pores with 10 families and $S R_{\max }=10$.
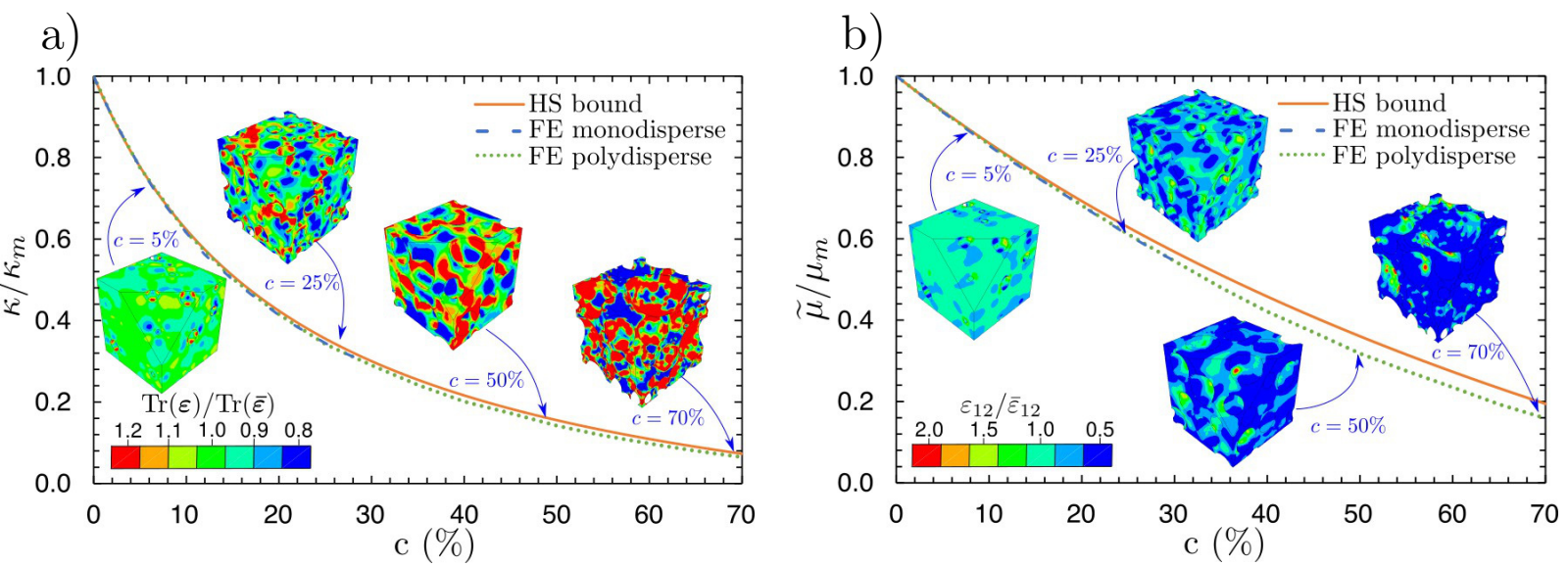

Figure 12: Normalized with the matrix properties effective (a) bulk modulus $\widetilde{\kappa} / \kappa_{m}$ and (b) shear modulus $\widetilde{\mu} / \mu_{m}$ as a function of the porosity $c$. Comparison between the analytical HS bounds, FE monodisperse numerical estimates and experimental results.

Using the periodic boundary conditions (PBC) discussed in Section 2.2, we estimate the effective elastic properties for polydisperse porosities $c \in[0,70 \%]$. The computed effective bulk and shear moduli are compared to the Hashin-Shtrikman upper bounds in Fig 12 as well as the previously recorded FE monodisperse estimates. In this figure, we observe a remarkably good agreement between the FE numerical estimates and the HS bounds for the entire range of porosities even for $70 \%$. The maximum difference is found to be in the order of $5 \%$ for the shear modulus at that maximum porosity. This result motivates further attempts to reach such high volume fractions experimentally, noting however that with such increase of porosity the interactions between the voids become even more important. As a consequence, the material between the 
inter-void spacing is expected to show higher strain fluctuations with respect to the average applied strain and hence more a pronounced nonlinear response in the real experiments.

\section{Concluding remarks}

In this work, we have presented numerically-aided additive manufacturing of random isotropic closedcell porous materials with controlled effective elastic properties. Specifically, the 3D printed specimens were realized by use of the random sequential adsorption method which was used to obtain random monodisperse spherical porous microstructures. A complete FE numerical study allowed to define a cubic representative volume element (RVE) by combined periodic and kinematically uniform (i.e. average strain or affine) boundary conditions. The resulting cubic RVE has been subsequently assembled to form a standard dogbone uniaxial tension specimen, which was 3D printed by use of a photopolymeric resin material. The specimen was then tested at relatively small strains (in the order of $0.7 \%$ nominal axial strain) by a proper multi-step relaxation procedure to obtain the purely energetic elastic moduli.

The 3D printed porous and non-porous specimens were extensively analyzed in order to address the questions of isotropy due to the printing procedure, as well as that due to the randomness of the microstructure. The overall experimental response of the RVEs was found to be isotropic and reproducible allowing for a direct comparison with the corresponding numerical estimates and the theoretical upper Hashin-Shtrikman bounds.

We have shown that the proposed random monodisperse microstructures lead to almost optimal effective elastic properties experimentally since they were shown to be in very close agreement (less than $8 \%$ ) with the Hashin-Shtrikman bounds for porosities up to 30\%. Even that difference could be attributed to various factors such as the experimental measurement uncertainty, statistical deviations due to the printing procedure and the RVEs. The possibility to extend the proposed RVEs to higher porosities has also been investigated numerically by use of polydisperse (i.e. different size) void microstructures, which were shown to lie very close to the Hashin-Shtrikman bounds even up to porosities of $70 \%$. The additive manufacturing of such polydisperse closed-cell high porosity RVEs is yet to be realized due to practical constraints during the $3 \mathrm{D}$ printing process. An effort to overcome those issues is in progress.

Finally, the great versatility of the random sequential addition algorithm allows to extend the generation process to anisotropic microstructures. Those microstructures could involve ellipsoidal voids randomly or unidirectionally dispersed in the RVE. They could be allowed to overlap or simply connected via cylindrical (throat-type) voids. These materials can also be used as test beds to a number of experimental procedures involving dynamic and static measurements of elastic moduli via wave dispersion and impact loads. Extension of the present study to the nonlinear regime (nonlinear elastic and viscoelastic (Lopez-Pamies et al., 2013) as well elasto-plastic (Mbiakop et al., 2015)) is also straightforward and is left for a future study. 
We close by noting that the present cubic RVEs can be assembled in more complex macrostructures with varying porosities at different regions allowing the optimization of both density and stiffness (Allaire, 2012).

\section{Acknowledgments}

The authors acknowledge support of the European Research Council (ERC) under the European Unions Horizon 2020 research and innovation program (grant agreement no. 636903, MAGNETO). O.Z. and K.D. also acknowledge support from TOTAL S.A. The computational part of this work was also supported by the ANR under contract number ANR-10-EQPX-37.

\section{References}

ABAQUS Version 6.11, 2011. Documentation. Dassault Systémes Simulia Corp. Providence, RI, USA.

Alderson, A., Rasburn, J., Ameer Beg, S., Mullarkey, P.G., Perrie, W., Evans, K.E., 2000. An auxetic filter: a tuneable filter displaying enhanced size selectivity or defouling properties. Industrial Engineering Chemistry Research 39, 654-665.

Allaire, G., 2012. Shape optimization by the homogenization method. Springer Science and Business Media.

Anoukou, K., Brenner, R., Hong, F., Pellerin, M., Danas, K., 2018. Random distribution of polydisperse ellipsoidal inclusions and homogenization estimates for porous elastic material. Computers and Structures in press.

Banhart, J., Baumeister, J., Weber, M., 1996. Damping properties of aluminium foams. Materials Science and Engineering: A $205,221-228$

Barclift, M.W., Williams, C., 2012. Examining variability in the mechanical properties of parts manufactured via polyjet direct 3D printing. 23rd Annual International Solid Freeform Fabrication Symposium - An Additive Manufacturing Conference, SFF 2012 .

Bendsoe, M.P., Ben-Tal, A., Zowe, J., 1994. Optimization methods for truss geometry and topology design. Structural optimization $7,141-159$.

Berger, J.B., Wadley, H.N.G., McMeeking, R.M., 2017. Mechanical metamaterials at the theoretical limit of isotropic elastic stiffness. Nature 543, $533 \mathrm{EP}-$.

Böhm, H., Eckschlager, A., Han, W., 2002. Multi-inclusion unit cell models for metal matrix composites with randomly oriented discontinuous reinforcements. Computational Materials Science 25, $42-53$.

Böhm, H.J., Han, W., 2001. Comparisons between three-dimensional and two-dimensional multi-particle unit cell models for particle reinforced metal matrix composites. Modelling and Simulation in Materials Science and Engineering 9, 47.

Bucataru, I., Slawinski, M.A., 2008. Invariant properties for finding distance in space of elasticity tensors. Journal of Elasticity 94, 97-114.

Cansizoglu, O., Harrysson, O., Cormier, D., West, H., Mahale, T., 2008. Properties of ti-6al-4v non-stochastic lattice structures fabricated via electron beam melting. Materials Science and Engineering: A 492, $468-474$.

Danas, K., 2017. Effective response of classical, auxetic and chiral magnetoelastic materials by use of a new variational principle. Journal of the Mechanics and Physics of Solids 105, $25-53$

Davies, G.J., Zhen, S., 1983. Metallic foams: their production, properties and applications. Journal of Materials Science 18, 1899-1911.

Deshpande, V., Fleck, N., 2000. Isotropic constitutive models for metallic foams. Journal of the Mechanics and Physics of Solids 48, $1253-1283$. 
Eshelby, J., 1957. The determination of elastic field of an ellipsoidal inclusion and related problems. Proceedings of the Royal Society of London , 379-396.

Fleck, N., Deshpande, V., Ashby, M., 2010. Micro-architectured materials: past, present and future. Proceedings of the Royal Society of London A: Mathematical, Physical and Engineering Sciences 466, 2495-2516.

Francfort, G.A., Murat, F., 1986. Homogenization and optimal bounds in linear elasticity. Archive for Rational Mechanics and Analysis 94, 307-334.

Ghossein, E., Lévesque, M., 2014. A comprehensive validation of analytical homogenization models: The case of ellipsoidal particles reinforced composites. Mechanics of Materials 75, $135-150$.

Gibson, L.J., Ashby, M.F., 1997. Cellular Solids: Structure and Properties. Cambridge Solid State Science Series, Cambridge University Press. 2 edition.

Göransson, P., 2006. Acoustic and vibrational damping in porous solids. Philosophical Transactions of the Royal Society of London A: Mathematical, Physical and Engineering Sciences 364, 89-108.

Gorny, B., Niendorf, T., Lackmann, J., Thoene, M., Troester, T., Maier, H., 2011. In situ characterization of the deformation and failure behavior of non-stochastic porous structures processed by selective laser melting. Materials Science and Engineering: A $528,7962-7967$.

Hashin, Z., 1962. The elastic moduli of heterogeneous materials. Journal of Applied Mechanics , 481-505.

Hashin, Z., Shtrikman, S., 1963. A variational approach to the theory of the elastic behaviour of multiphase materials. Journal of the Mechanics and Physics of Solids , 127-140.

Heinl, P., Rottmair, A., Körner, C., F. Singer, R., 2007. Cellular titanium by selective electron beam melting. Advanced Engineering Materials 9, $360-364$.

Hengsbach, S., Lantada, A.D., 2014. Direct laser writing of auxetic structures: present capabilities and challenges. Smart Materials and Structures 23, 085033.

Hill, R., 1952. The elastic behaviour of a crystalline aggregate. Proceedings of the Physical Society. Section A $65,349$.

Hill, R., 1963. Elastic properties of reinforced solids: Some theoretical principles. Journal of the Mechanics and Physics of Solids $11,357-372$.

Hossain, M., Vu, D., Steinmann, P., 2012. Experimental study and numerical modelling of vhb 4910 polymer. Computational Materials Science 59, 65-74.

Huet, C., 1990. Application of variational concepts to size effects in elastic heterogeneous bodies. Journal of the Mechanics and Physics of Solids 38, $813-841$.

Idiart, M.I., 2008. Modeling the macroscopic behavior of two-phase nonlinear composites by infinite-rank laminates. Journal of the Mechanics and Physics of Solids 56, $2599-2617$.

Jeulin, D., 2001. Random structure models for homogenization and fracture statistics. D. Jeulin, M. Ostoja-Starzewski (Eds.), Mechanics of Random and Multiscale Microstructures, Springer-Verlag.

Kanit, T., Forest, S., Galliet, I., Mounoury, V., Jeulin, D., 2003. Determination of the size of the representative volume element for random composites: statistical and numerical approach. International Journal of Solids and Structures 40,3647 - 3679.

Lopez-Pamies, O., Goudarzi, T., Danas, K., 2013. The nonlinear elastic response of suspensions of rigid inclusions in rubber: $\mathrm{Ii}$ - a simple explicit approximation for finite-concentration suspensions. Journal of the Mechanics and Physics of Solids 61 , $19-37$.

Ma, Y., Scarpa, F., Zhang, D., Zhu, B., Chen, L., Hong, J., 2013. A nonlinear auxetic structural vibration damper with metal rubber particles. Smart Materials and Structures 22, 084012.

Mbiakop, A., Constantinescu, A., Danas, K., 2015. An analytical model for porous single crystals with ellipsoidal voids. Journal of the Mechanics and Physics of Solids 84, $436-467$.

Messner, M., 2016. Optimal lattice-structured materials. Journal of Mechanics Physics of Solids 96, $162-183$. 
Meza, L., Das, S., Greer, J., 2014. Strong, lightweight, and recoverable three-dimensional ceramic nanolattices. Science 345, $1322-1326$.

Meza, L.R., Phlipot, G.P., Portela, C.M., Maggi, A., Montemayor, L.C., Comella, A., Kochmann, D.M., Greer, J.R., 2017. Reexamining the mechanical property space of three-dimensional lattice architectures. Acta Materialia 140,424 - 432.

Moakher, M., Norris, A.N., 2006. The closest elastic tensor of arbitrary symmetry to an elasticity tensor of lower symmetry. Journal of Elasticity 85, 215-263.

Moussaddy, H., Therriault, D., Lévesque, M., 2013. Assessment of existing and introduction of a new and robust efficient definition of the representative volume element. International Journal of Solids and Structures $50,3817-3828$.

Queheillalt, D.T., Murty, Y., Wadley, H.N., 2008. Mechanical properties of an extruded pyramidal lattice truss sandwich structure. Scripta Materialia 58, $76-79$.

Rintoul, M., Torquato, S., 1997. Reconstruction of the structure of dispersions. Journal of Colloid and Interface Science 186, $467-476$.

Schöberl, J., 1997. Netgen an advancing front 2d/3d-mesh generator based on abstract rules. Computing and Visualization in Science 1, 41-52.

Segurado, J., Llorca, J., 2002. A numerical approximation to the elastic properties of sphere-reinforced composites. Journal of the Mechanics and Physics of Solids 50.

Sigmund, O., 2000. New class of extremal composites. Journal of the Mechanics and Physics of Solids 48, 397-428.

Spoor, P.S., Maynard, J.D., Kortan, A.R., 1995. Elastic isotropy and anisotropy in quasicrystalline and cubic alculi. Phys. Rev. Lett. 75, 3462-3465.

Suquet, P., 1987. Homogenization Techniques for Composite Media: Lectures Delivered at the CISM International Center for Mechanical Sciences Udine, Italy, July 1-5, 1985. Springer Berlin Heidelberg, Berlin, Heidelberg. pp. $193-230$.

Sypeck, D.J., 2005. Cellular truss core sandwich structures. Applied Composite Materials 12, 229-246.

Tancogne-Dejean, T., Mohr, D., 2017. Elastically-isotropic truss lattice materials of reduced plastic anisotropy. International Journal of Solids and Structures .

Torquato, S., 2002. Random Heterogeneous Materials: Microstructure and Macroscopic Properties. Springer, New York.

Wang, D.W., Li, F., Liu, M., Lu, G., Cheng, H.M., 2008. 3d aperiodic hierarchical porous graphitic carbon material for high rate electrochemical capacitive energy storage. Angewandte Chemie International Edition 47, 373-376.

Willis, J., 1977. Bounds and self-consistent estimates for the overall properties of anisotropic composites. Journal of the Mechanics and Physics of Solids 25, $185-202$.

Wissler, M., Mazza, E., 2007. Mechanical behavior of an acrylic elastomer used in dielectric elastomer actuators. Sensors and Actuators A: Physical 134, $494-504$.

Zener, C.M., Siegel, S., 1949. Elasticity and anelasticity of metals. The Journal of Physical and Colloid Chemistry 53, $1468-1468$.

Zok, F., Waltner, S., Wei, Z., Rathbun, H., McMeeking, R., Evans, A., 2004. A protocol for characterizing the structural performance of metallic sandwich panels: application to pyramidal truss cores. International Journal of Solids and Structures $41,6249-6271$. 